\title{
Comte revu et corrigé : le cas Littré
}

Comte's sociology revised : the case of Littré

\section{Annie Petit}

\section{(2) OpenEdition}

\section{Journals}

Édition électronique

URL : http://journals.openedition.org/ress/3591

DOI : 10.4000/ress.3591

ISBN : 1663-4446

ISSN : 1663-4446

\section{Éditeur}

Librairie Droz

Édition imprimée

Date de publication : 30 novembre 2016

Pagination : 69-88

ISSN : 0048-8046

\section{Référence électronique}

Annie Petit, «Comte revu et corrigé : le cas Littré », Revue européenne des sciences sociales [En ligne] 54-2 | 2016, mis en ligne le 30 novembre 2019, consulté le 02 janvier 2020. URL : http:// journals.openedition.org/ress/3591; DOI : 10.4000/ress.3591 


\title{
COMTE REVU ET CORRIGÉ: LE CAS LITTRÉ
}

\author{
ANNIE PETIT \\ Université Montpellier 3 \\ anniepetit2@wanadoo.fr
}

Résumé. On analyse ici les positions d'Émile Littré, l'un des disciples les plus proches d'Auguste Comte, mais aussi l'un des plus infidèles, à propos de la sociologie. Après un rappel du cadre et des limites de son allégeance, on examine son premier militantisme où l'on décèle déjà les thèmes des différends ultérieurs. Ils touchent surtout à la compréhension d'une science sociale avant tout liée à la politique actuelle et à la confusion de la sociologie et l'histoire. Après s'être écarté de Comte, Littré développe une sociologie qui accentue encore ces divergences. Cette question des liens entre sociologie, politique et histoire hantent les débats de ceux qui, en France, s'efforcent d'institutionnaliser la nouvelle discipline.

Mots-clés: Auguste Comte, histoire, Émile Littré, positivisme, sociologie.

\begin{abstract}
This article deals with Émile Littré's views on sociology. Littré was one of Auguste Comte's closest but also one of his most unfaithful followers. After analyzing the framework and limits of his allegiance, I focus on his early positivist commitment, where the first signs of future disagreements can already be detected. These mainly relate to a conception of social science as being primarily linked with current politics, as well as to the conflation of sociology with history. Once emancipated from Comte, Littré develops a sociology which further emphasized these disagreements. These issues bearing on the relations between sociology, politics and history were recurrent in the French debates concerning the institutionalization of the new discipline.
\end{abstract}

Keywords: Auguste Comte, history, Émile Littré, positivism, sociology. 
Auguste Comte est à la recherche depuis sa jeunesse d'une «physique sociale» qu’il fonde dans le Cours de philosophie positive en lui donnant en I839 le nom de «sociologie». Émile Littré est, lorsqu'il lit le Cours en I840, un auteur déjà connu auquel sont ouverts de nombreux journaux et revues'. Il s'enthousiasme pour la nouvelle philosophie et en fait la promotion dans Le National. Par la suite, Littré se montre à la fois très proche de Comte et volontiers infidèle, au point de quitter dès 1852 la Société positiviste qu'il avait contribué à fonder en mars I $848^{2}$. Il s’agit ici d'examiner de plus près les positions de ce disciple indiscipliné, en particulier pour ce qui touche à la philosophie des sciences sociales.

\section{CADRES ET LIMITES D’UNE ALLÉGEANCE}

Littré entre en relation avec Comte en I844. Les articles qu'il lui consacre dans Le National à partir du mois de novembre assurent une importante audience à Comte, lequel est ravi de cette efficace recrue et juge Littré comme «l'homme de France qui a le plus complètement saisi et apprécié l'ensemble de la nouvelle philosophie » (Comte, I973-1990, t. 2, p. 292-lettre à J. S. Mill du 2 I octobre i844). Littré devient bientôt l'un des membres plus actifs de la Société positiviste : il en rédige des rapports importants et est l'inventeur du subside au maître. Lors de la fondation de cette même Société, Comte impose aux membres de connaître et admettre les thèses positives dont ils peuvent prendre connaissance, selon lui, soit par le Cours de philosophie positive, soit par le Discours sur l'esprit positif, soit encore par «l'éminent opuscule De la philosophie positive de Littré» (Littré, I845a), qu’il inscrit, en 1851, dans les ouvrages sélectionnés pour la première «Bibliothèque positiviste». À cette date, Comte voit toujours en Littré un «positiviste d'élite» (Comte, I973-1990, t. 6, p. I8I).

I Littré avait déjà entrepris (en 1839) l'édition des Fuvres complètes d'Hippocrate (il la terminera en 186I, chez Baillière: 10 volumes) et publié de nombreux articles.

2 Voir sur ces relations complexes, Annie Petit, 1993, vol.2, chap. 8, et Mary Pickering, 1993-2009, vol. 2. 
Mais les rapports se gâtent entre les deux hommes et des tensions se font jour en matière politique. Comte admet le coup d'état de Louis-Napoléon Bonaparte, espérant qu'il sera l'homme fort capable d'imposer une nouvelle direction politique conforme aux vœux positivistes (Petit, 1987). Littré, ardemment républicain, n’apprécie guère. De plus, il n’apprécie pas non plus les tournures religieuses du positivisme comtien et la manière dont Comte les met sous l'inspiration de son amour pour Clotilde de Vaux³. Dès janvier I852, Littré quitte la Société positiviste, tandis que Comte l'en «excommunie» en août, déclarant avec grand fracas la «rupture irrévocable». Littré est dès lors jugé non seulement comme un «positiviste intellectuel» et «positiviste incomplet», mais aussi comme «déserteur», «pédant académique», «lâche écrivain » et «érudit épuisé » (voir Comte, I973-1990, t. 6, p. 313-345). L’intéressé adopte une conduite délibérément apaisante et retourne à ses études médicales et philologiques. Il persiste pourtant à considérer Comte comme son «maître » et à s'en dire «disciple $»^{5}$; puis, après la mort de Comte, Littré prend très vite la stature de chef du mouvement positiviste ${ }^{6}$.

Cependant Littré est tout à fait précis au sujet de son affiliation et sur ses limites. Le positivisme dont il se réclame est celui du Cours, non pas celui du Système. Les titres mêmes de ses ouvrages militants sont révélateurs car ils se réfèrent à la «philosophie positive», évitant la mention au «positivisme» dont le maître

3 Pourtant Littré a souscrit aux premiers développements «religieux»: voir ses articles sur la religion de l'Humanité au National, «Idéal ou religion» du 10 septembre |849, «Idée religieuse de l'Humanité» du 10 mars 1851 et «Théorie positive de la révélation et de la félicité» du 14 avril I85I (Littré, 1852, p. 122-130 et 287-295).

4 Les «vrais positivistes» sont ceux qui souscrivent au «positivisme religieux»: ce distinguo est fréquemment employé par Comte dans sa correspondance d'après 1852. Voir aussi ses jugements sur «l'inconséquence des partisans intellectuels du positivisme» dans sa «Préface spéciale de l'Appendice» de 1969-1970 [1851-1854], vol.4, p.II - texte que Littré reprend et discute (voir 1863, «Conclusion»).

5 Littré continue d'ailleurs à verser le subside au Grand-Prêtre d'une religion qu'il ne reconnaît pourtant pas. Sur sa revendication comme disciple, voir sa «Préface d'un disciple» (I864). Voir aussi comment Littré (1867) défend au besoin Comte contre Mill.

6 En fait, Littré et ses amis de la La Philosophie positive sont nettement plus connus et influents que les positivistes du groupe «orthodoxe», dirigé par Pierre Laffitte, qui n'aura réellement d'influence qu'après la mort de Littré. 
avait bien dit qu'il comprenait «une philosophie et une politique» (Comte, I969I970 [I85I-I854], vol. I, p. 2)7. Littré n’use des termes «positivisme» ou «positiviste» quavec retenue. Ainsi, c'est le titre Paroles de philosophie positive qu'il choisit pour réunir certains de ses articles (Littré, I859 ${ }^{8}$ ). Son volumineux ouvrage de I863 est intitulé Auguste Comte et la philosophie positive; et lorsqu'il fonde en I867 la première revue expressément liée à la philosophie de Comte, il l'appelle significativement La Philosophie positive. Le terme «positivisme» n’apparaît en fait que dans un seul titre de Littré, Conservation, Révolution, Positivisme, de I852, qui reprend les articles du National dont un seul inscrit dans son titre le «positivisme»; or ce livre est précisément celui à propos duquel Comte et Littré commencèrent à prendre la mesure de leurs différends`. Dans son Dictionnaire de la langue française, d’ailleurs, c'est en tant que «système de la philosophie positive» que Littré inscrit le terme «positivisme». Certes, il ne le proscrit pas et le cautionne même dans quelques textes, mais tardivement, comme lorsqu'il fait paraître une «Étude sur les progrès du positivisme», jugés satisfaisants (Littré, I $877^{\circ \circ}$ ). Il n'en demeure pas moins que le «positivisme» du disciple reste fort différent de ce que le maître avait nommé ainsi et espéré.

Littré est ainsi à la fois celui qui a travaillé à la promotion de la pensée de Comte, mais aussi celui qui a accrédité la thèse d'une dualité-contradiction dans l’œuvre de ce dernier. Pour lui, Comte a été inconséquent dans sa méthode ${ }^{11}$. Se jugeant en demeure de choisir, c'est au nom même de la méthode positive, et pour la sauver, que Littré entreprend donc un travail de tri. Il a dû, précise-t-il,

7 Ce sont les premiers mots du Préambule général du «Discours préliminaire » du Système de politique positive.

8 Republié en 1863 chez Ladrange, cet opuscule (6I p.) est réédité aussi dans Littré, 1876, p.90-132.

9 Littré (1879a) en fera une réédition enrichie de nombreuses «remarques» autocritiques.

10 Ce texte est publié aussi dans la $4^{\mathrm{e}}$ édition du Cours de philosophie positive (Baillière, 1877), après la «Préface d'un disciple» (1864) qui ouvrait la $2^{\mathrm{e}}$ édition.

II Voir Littré, |863, «Préface», p.II-V: «M. Comte, à un moment donné, pensant et assurant qu'il ne faisait que développer la philosophie positive, changea de méthode [...]. II fut impossible de trouver cette conséquence que $M$. Comte avait affirmée; les deux parties se montrèrent comme deux doctrines distinctes, ayant des points différents et inconciliables [...]. De la sorte, maintenant avec fermeté la philosophie positive qui est la base, j'ai, avec non moins de fermeté, rejeté pour une grande part, la politique positive que $M$. Comte a voulu en déduire». 
«scinder M. Comte» (Littré, I863, p. IV) ${ }^{12}$. Le système est bon, à condition d'élaguer certaines implications et applications que Comte a cru cohérentes, mais qui ne sont aux yeux de Littré que des dérives pathologiques ${ }^{13}$. Et toutes relèvent du fait que Comte aurait, avec inconséquence, échangé la méthode objective pour une méthode subjective insoutenable (1863, p. III-V, p. 517-519, p. 669-670). Dans la $3^{\mathrm{e}}$ partie du même ouvrage, Littré développe et assume des divergences plus ou moins profondes; enfin la conclusion recense les «lacunes » du maître: économie politique, théorie cérébrale, et ce que Littré appelle «théorie subjective de l'humanité » - revendication très large (ibid., p. 677-678). Les différends alors exprimés sont graves: ils concernent rien de moins que la classification des sciences et l'ordonnance de l'avenir des recherches.

Venons-en à présent à l'examen des positions de Littré dans le domaine de la philosophie de la sociologie et de l'histoire. Car, en dépit de ses réserves sur le Système de politique positive ou Traité de sociologie, Littré s'est beaucoup intéressé à ce domaine promu par Comte en nouvelle discipline scientifique. J’ai déjà étudié précédemment quelques aspects des débats entre Comte et Littré (Petit, I992a et 1992b); il s'agit ici d’approfondir l'analyse en l'insérant mieux dans une histoire des conceptions de la sociologie.

12 «Je n'ai point eu à scinder l'œuvre de M. Comte, qui reste intacte et entière; je n'ai eu qu'à en retrancher des conséquences et des applications impropres. Mais j'ai eu, et cela a été douloureux, à scinder M. Comte lui-même, c'est-à-dire à montrer que, quand il a voulu passer des principes posés dans le système de philosophie positive à l'application posée dans la politique positive, il n'a pas tenu d'une main sûre le fil qui devait le conduire». Voir aussi Littré, I863, p.519: «Heureusement c'est toujours avec M. Comte, avec la méthode créée par lui que je signale les erreurs contre la méthode». On peut enfin se reporter à toute la «Conclusion» du livre, surtout p.668-67I.

13 Littré médecin vient ainsi au secours du Littré philosophe lorsqu'il s'agit d'invoquer d'éventuels effets pathologiques: surmenage et passion entraînant une «grave crise nerveuse», le genre de «secousses mentales modifiant grandement les dispositions intérieures» (ibid., p. V, p. 58I-584). L'«hygiène mentale» de Comte est jugée par Littré un fort mauvais régime, causant, ou/et causé par, un mysticisme galopant (ibid., p.586-589). Même principe de critique lorsque Littré déplore l'emploi par Mill du terme «ridicule» pour qualifier certains des derniers propos de Comte: «Ces absurdités sont plutôt pathologiques que philosophiques» (I867, p.7). 


\section{LE MILITANTISME DE LITTRÉ AU NATIONAL}

Dès les articles du National, on voit combien Littré est fasciné par le programme sociologique de Comte. Car c'est bien, plutôt que sur les apports d'une philosophie générale des sciences, sur les aspects socio-politiques de la doctrine comtienne que que Littré met l'accent.

La première série d'articles (novembre-décembre I844) est celle réunie dans De la philosophie positive (1845a) ${ }^{14}$. Littré y fait une présentation générale de la nouvelle philosophie qui, selon lui, est la seule apte à organiser les savoirs. Il commence par exposer l'innovation que représente la façon dont Comte aborde les phénomènes sociaux et précise, entre autres, les traits de la méthode proposée; puis il analyse le rapport de cette philosophie à l'ensemble des sciences. Littré fait surtout gloire à Comte d'avoir cherché, et su trouver, des lois dans l'histoire alors que des esprits moins perspicaces n'y voient qu'indéterminations et incohérence. Cette conversion du regard historique, fondatrice de la «science sociale», est une véritable révolution intellectuelle assurant l'alliance, ou, mieux encore, la fusion de la philosophie et des sciences (I852, p. 48-66 notamment). On soulignera que, après avoir récapitulé les points capitaux de l'œuvre comtienne, les derniers mots de premier ensemble sont pour insister sur son inscription dans l'histoire (ibid., p. 66).

Les deux autres séries d’articles relèvent de la réflexion politique engagée dans l'esprit post-quarante-huitard, l'importance de la dernière commotion révolutionnaire donnant un regain d'intérêt aux questions sociales.

La deuxième série comprend onze articles parus entre juillet et octobre I849. Regroupés sous le titre Application de la philosophie positive au gouvernement des sociétés et en particulier à la crise actuelle ${ }^{15}$, ils proposent une analyse de la Révolution, de ses conditions, et dressent le bilan de ce qui est fait tout en programmant ce qu'il reste à faire. L'orthodoxie de ces textes se veut rigoureuse. Littré cite fréquemment Comte, loue son «jugement si clairvoyant», ses «lumineuses conceptions», son «génie» (I852, p.68, p. 72, p. 8I). Le premier article pose «le problème de la

I4 Publié également sous un autre titre (1845b), ce texte fut repris quatre fois par la suite, la dernière dans Littré, 1876.

15 Publiés en ouvrage en 1850 (Paris, Ladrange), ils forment la $2^{\mathrm{e}}$ partie de Littré, 1852. 
rénovation totale et définitive qui doit clore la révolution » (ibid., p. 74). Les autres montrent ensuite, de différents points de vue, que seule la philosophie positive peut le résoudre et s'appuient sur des analyses tout à fait conformes à celles du Cours. Puis, ayant ainsi exposé les bases scientifiques d'un nouvel ordre social, Littré reprend les projets de réorganisation présentés par la Société positiviste ${ }^{16}$. La conclusion est un éloge de la sociologie comtienne et les derniers mots approuvent la nécessité de l’appuyer sur une «religion de l'Humanité» (ibid., p. I67-168).

La troisième série (juin I850-octobre 1851) comprend vingt articles. Dix examinent le «côté négatif», c'est-à-dire les mesures prises pour essayer d’arrêter le désordre; dix autres présentent le «côté positif», c'est-à-dire les efforts faits pour constituer un nouvel ordre ${ }^{17}$. Il s'agit de textes assez disparates. D’abord un Préambule, véritable profession de foi socialiste, républicaine et pro-prolétarienne ${ }^{18}$. Suivent des analyses circonstanciées, à propos de telle ou telle mesure de politique contemporaine, qui sont l'occasion de rappeler les propositions positivistes. Puis Littré reprend des propos plus généraux aboutissant à un prosélytisme actifi. Le positivisme est ainsi présenté comme une variété, mieux, comme la vérité, du socialisme: la philosophie positive est la seule capable de le «déterminer », de lui donner une «issue $»^{20}$. Peu à peu épuré des tendances révolutionnaires, le socialisme est transformé en propédeutique, en vrai «parti de

I6 Réforme de l'éducation et de l'instruction, séparation des pouvoirs temporel et spirituel, organisation d'une nouvelle culture, restructuration des rapports internationaux, révision de la Constitution pour installer un gouvernement prolétarien provisoire capable de gérer «la fin de l'état révolutionnaire» et de prendre fermement des mesures prochaines: réduction de l'armée, suppression du budget des cultes et de l'Université, etc.

17 Ces articles sont republiés pour la première fois dans Littré, 1852, 3e partie, sous le titre «Des progrès du socialisme».

I8 Le socialisme y est présenté comme étant le couronnement-dépassement de la Révolution (ibid., p. 169-177). Dans la précédente série (Application) Littré avait déjà consacré un article au «Socialisme», défini par rapport à la révolution (ibid., p. 93).

19 «Nous, socialistes», dit plusieurs fois Littré (ibid., p. 173, p. 228, p. 229, etc.). Ce militantisme se spécifie dans un emboîtement de déterminations: «le positiviste renferme le socialiste, le socialiste renferme le républicain, et le républicain renferme le libéral» (ibid., p. 256).

20 Voir les articles «Socialisme indéterminé» et «Du Socialisme déterminé ou philosophie positive »: «J'appelle socialisme indéterminé, tout système socialiste qui s'occupe particulièrement de régler l'ordre temporel et qui laisse de côté l'ordre spirituel » (ibid., p. 263). 
l'ordre » dont Littré attend tous les progrès, à condition de ne point le confondre avec le parti conservateur qui prétend s'en faire le spécialiste. Cette dernière série d’articles se termine encore sur l’affirmation des liens entre théorie sociale, programme politique et promotion d'une nouvelle religion:

Le socialisme intervient comme un anneau entre ce passé qui se dissout et la notion suprême de l'Humanité, qui sera la religion de l'avenir [...]. Le développement naturel de l'histoire vient en aide au socialisme pour lui ouvrir l'issue qui lui manque. La religion révélée touche à son terme [...]. La religion démontrée vient prendre sa place; les sciences ont défait toute théologie; mais transformées en une seule science ou philosophie, elles refont une nouvelle base religieuse pour la société de l'avenir. Cette base, c'est l'Humanité (ibid., p. 326-327).

Toute la collaboration de Littré au National montre une grande fidélité aux thèses comtiennes; mais, par-delà les accords reconnus ${ }^{21}$, les positions de Littré sont assez décalées, notamment sur le statut - place et méthode - de la «sociologie ${ }^{22}$. En effet, certains points sont porteurs de divergences futures. Deux surtout.

L’un est plutôt général. Dans ce premier exposé de la philosophie positive, l'ordre adopté par Littré est original par rapport à l'ordre des leçons comtiennes. Il présente «d’abord la théorie sociale, puis la philosophie qui sort de l'ensemble des sciences définitivement complétées » (Littré, I852, p. 7), c'est-à-dire d’abord la partie de l'ouvrage de Comte consacrée aux phénomènes sociaux, avant d’analyser les différences des approches théologiques ou métaphysiques et positives ainsi que l'ordonnance des sciences. Littré inverse ainsi l'ordre même des progrès du Cours, soulignant combien la «science sociale» est, pour lui, le lieu déterminant de l'élaboration de la philosophie positive ${ }^{23}$. Certes, Comte lui-même a dit l'enracinement de toute sa pensée dans une visée de réorganisation sociale ; mais Littré

21 Voir Comte déclarant à Mill, le 25 décembre 1844: «je ne pouvais désirer une appréciation plus complète et plus profonde, à tous égards plus satisfaisante». Mill est de cet avis dans sa réponse à Comte du 31 décembre (Comte, 1973-1990, t.2, p. 303 et p.430).

22 Comte d'ailleurs prétend bientôt, tout en continuant à louer la première série d'articles, que le recueil de 1852 manque de cohérence. Voir ses lettres à Georges Audiffrent, du 6 juillet, et à Alexandre Laurent, du 14 septembre 1852 (ibid., t. 6, p. 313 et p. 364-365).

23 Voir aussi sa présentation du statut de la «science sociale» dans le dernier article sur l'ensemble de la philosophie positive et la hiérarchie des sciences, ibid., p. 62-65. 
recentre nettement son attention sur les conséquences pratiques qu’il est permis de tirer de la connaissance des phénomènes sociaux.

L'autre point de divergence en découle et, apparemment plus particulier, il est en fait lourd d'implications. Il y a chez Littré traduction voire confusion constante entre la sociologie et l'histoire. Ainsi exprime-t-il le programme de la philosophie positive dans son premier article :

Faire de l'histoire une science et créer une philosophie positive, sont deux idées consécutives, mais connexes, et qui, au point où est arrivée l'humanité, ne peuvent être séparées. Faire de l'histoire une science (l'histoire n'est que la société considérée dans le temps), c'est d'une part reconnaître que les phénomènes sociaux se suivent dans une succession qui n'est ni arbitraire ni fortuite, et d'autre part déterminer la loi de cette succession; tant que ce résultat n'est pas obtenu, ou bien les faits, à l'état de simples matériaux, ne sont qu'objets d'érudition, ou bien ils se prêtent à toutes les explications, quelques vagues qu'elles soient; et c'est la double condition dans laquelle l'histoire est encore aujourd'hui (ibid., p. 6).

Et Littré d’en référer au «temps, jusqu'ici seul instructeur en fait de notions historiques et politiques», d’identifier «les événements historiques, c'est-à-dire les phénomènes sociaux», et de rapporter l'essentiel de la philosophie comtienne à une exploration de l'histoire (ibid., p. 13, p. 15). Le second article assume, dans son titre même, l'identification constamment suggérée par le premier : «De la science sociale, ou science de l'histoire »; et partout Littré appuie ses analyses des thèses de Comte sur le «tableau de l'histoire», «les leçons de l'histoire»:

Rien de plus instructif que ce contraste fourni par l'histoire, maintenant suffisamment étendue pour que l'expérience soit concluante. Le temps laisse sourdre peu à peu ses enseignements comme autant de minces filets d'eau qui sillonnent à peine le sol; mais à la longue ces filets réunis forment un courant qui entraîne les intelligences (ibid., p. 45).

Il est de plus à souligner que dans les articles de 1844 Littré n’emploie jamais le néologisme comtien de «sociologie» - ni même l'expression «physique sociale»- lui préférant les expressions «science sociale», «science de l'histoire », «théories des sociétés ». On ne trouve l'emploi du terme «sociologie» par Littré qu'à partir des articles de la deuxième série, et toujours renvoyé à 
l'histoire comme synonyme ${ }^{24}$. L'appel à l'histoire est aussi ce que Littré sélectionne volontiers dans les deux autres groupes d’articles, où est développée l'idée d'une assimilation parfaite entre l'histoire et la sociologie ${ }^{25}$.

Ce différend entre Comte et Littré est profond et n’échappe pas au premier qui proteste presque tout de suite:

La rédaction de la circulaire me semble tout à fait convenable. Je n'y désire qu'une légère rectification, qui est purement philosophique. Dans l'énumération hiérarchique des six sciences fondamentales, la dernière s'y trouve qualifiée d'histoire. Si vous le permettez, je substituerai ma dénomination ordinaire de sociologie comme étant non-seulement plus systématique, mais aussi plus complète, puisqu'elle embrasse à la fois l'étude statique et dynamique des sociétés, tandis que l'histoire ne convient qu'à la dynamique sociale (Comte, 1973-1990, t. 4, p. 207 - Lettre de Comte à Littré du I3 novembre I848).

Alors que pour Comte l'histoire est la «méthode» de la sociologie, donc une partie ou plutôt son outil, Littré persiste à les identifier. En se proclamant fondateur d'une science baptisée «sociologie», Comte en revendiquait une entière nouveauté. Littré la gomme quelque peu en s'en tenant à une science sociale-histoire. Et, loin d'insister, comme Comte, sur la dimension abstraite de la nouvelle science, Littré, en lui donnant principalement pour objet le règlement de la «crise actuelle», la présente plutôt pour ses «applications » concrètes, comme le confirment les titres choisis pour exposer les apports comtiens comme Application de la philosophie positive au gouvernement des sociétés, et en particulier à la crise actuelle ${ }^{26}$ ou comme participant aux « Progrès du socialisme ${ }^{27}$ ».

24 Voir ibid., p. 72: «la sociologie, ou histoire, ou science sociale comme on voudra l'appeler».

25 Dans l'article du 22 novembre 1844 du National, la formule comtienne «faire de la politique une science» est traduite par Littré: «faire de l'histoire une science». Dans ceux des 3 et 4 décembre, qui évoquent à plusieurs reprises la hiérarchie comtienne des six sciences, Littré persiste à appeler «histoire» la dernière science. Voir aussi le troisième article de la seconde série, «Progrès parallèle de la société et de la science, et destination sociale du positivisme», d'août |849, qui précise combien la philosophie positive est liée à l'étude de l'histoire, tant par l'explication qu'elle peut donner des filiations des états sociaux, que par l'efficacité de son ancrage dans la tradition historique.

26 1852, titre de la lle partie.

27 1852, titre de la III ${ }^{\text {e }}$ partie. 
Ainsi, au temps même de leur meilleure entente, il y avait bien des décalages dans les conceptions des deux auteurs.

\section{LA SOCIOLOGIE DE LITTRÉ}

Une fois la rupture consommée, Littré continue à faire l'équivalence entre histoire et sociologie, avec une telle persévérance qu'on ne peut s'empêcher d’y voir une stratégie de remaniement profond des choix comtiens. La subversion se déroule selon trois tactiques: Littré procède à des inflexions, qui invitent bientôt à des dissociations, et culminent dans une réinterprétation-appropriation proclamant de fortes divergences. Dans ses Paroles de philosophie positive, Littré nomme toujours la dernière des sciences positives plus volontiers «histoire » que «sociologie» et il ne dit guère «sociologie» sans prendre soin de préciser «ou histoire» (Littré, I859, p. I7 et p. 43). Puis, dans Auguste Comte et la philosophie positive, il ne saurait être plus explicite: «Les choses sociologiques sont si complexes que je n’aime pas les considérer indépendamment de l'histoire» (I863, p. 5I). Il fait d'ailleurs de Comte rien de moins qu'une sorte de Newton de l'histoire philosophique, celui qu'attendait Kant et qu'ont préparé Turgot et Condorcet (ibid., $\mathrm{I}^{\mathrm{re}}$ partie, chap. $3^{-} 6^{28}$ ); et, lorsqu'il défend une «méthode déductive» en sociologie, c'est pour mieux la rabattre sur l'histoire (ibid., $3^{\mathrm{e}}$ partie, chap. ${ }^{29}$ ). En I866, Littré assure que si Comte a tous «les droits [...] à se dire le constituteur de la sociologie», c'est en tant qu'il a découvert «la théorie du développement historique » (I867, p. 22-25). Plus tard, dans sa défense - bien comtienne - de l'autonomie de la sociologie par rapport à la biologie, Littré soutient encore que la sociologie n’a qu'un moyen pour saisir ce «fait fondamental» qu'est l'évolution de l'Humanité, à savoir l'histoire et l'étude du passé (I868, p. I86-207º). Et, en I870, dans un texte qui est comme une sorte de discours de la méthode en

Voir également p. 5I-52: «Les trois volumes qui terminent le Système de philosophie positive contiennent non une sociologie, mais le dessin du développement de l'histoire».

29 Littré y reproche à Comte d'avoir prôné, dans son Système, la méthode subjective «au lieu de s'en tenir à la méthode positive qui doit être déductive. [...] la méthode déductive en sociologie a justement pour l'avenir de l'histoire la même vertu que pour le passé [...] elle achève la philosophie de l'histoire et en assure les lois.» (p. 536-537).

30 Article repris dans Littré, I873, chap. XII. 
sociologie, Littré insiste tant sur ses complexités, qu'il en vient à «refuse[r] absolument» l'un des traits que Comte voulait faire assumer à la sociologie : l'aspect prédictif. Littré s'en tient donc à l'analyse historique et il n’hésite pas à se distancier des analyses comtiennes en appelant à les «modifier notablement», et insistant sur l'incertitude de toute prévision (I870, p. 297-30I ${ }^{31}$. Cette réticence de Littré à faire de la sociologie une discipline prédictive devient le mode même de son approche «sociologique». L'identification de la dernière science de la classification comtienne à l'histoire reste par la suite une constante des textes de Littré3².

Cependant, tout en maintenant ses différends, Littré a milité ardemment, à sa façon, pour un développement de la sociologie.

D’une part, il introduit le mot dans son Dictionnaire. La définition est «science du développement et de la constitution des sociétés humaines », et la création du vocable est attribuée à Comte ${ }^{33}$.

D’autre part, la sociologie lui paraît si importante qu'il institue, en I872, sous sa présidence, une Société de sociologie. Les statuts, avec la liste des membres fondateurs et la composition du bureau, paraissent aussitôt dans la revue La Philosophie positive, où sont aussi publiés les «quatre mémoires lus à la séance du 8 février i872, touchant la question de la classification des éléments de la sociologie, et qui a été la première mise à l'ordre du jour» (Littré, I872a, p. 298 et suiv.). L’histoire de cette société a fait l’objet de quelques études (voir Worms, I9Io et Heilbron, 2007) ; Johan Heilbron en a bien montré le fonctionnement, le type de réseaux sur lesquels elle s’appuie, le champ intellectuel «polarisé» dans lequel elle prend place et il propose d’intéressantes interprétations de sa fin précoce.

31 Après un nouveau balayage historique «depuis 1815 jusqu'à 1870》, l'article conclut: «Cela est incontestablement meilleur que le passé, mais cela reste toujours frappé de cette grande incertitude qu'on nomme le provisoire», ibid., p. 301.

32 Voir la classification présentée dans Littré, 1873 (p. I-VIII), où la dernière science est nommée «Histoire et sociologie».

33 Voir |863-|873, entrée «Sociologie». À signaler qu'il y avait eu un article «Sociologie» dans la $10^{e}$ édition du Dictionnaire de médecine, de chirurgie, de pharmacie, etc., de Pierre H. Nysten (Paris, Baillière) entièrement refondue par Littré et Charles Robin en 1855. L'article en référait expressément à Comte et à sa «série scientifique» dont la sociologie était présentée comme «la tête, le couronnement». 
Dans la perspective d'analyse philosophique adoptée ici, on reprendra cependant quelques points afin de mieux souligner les écarts entre la sociologie telle que l'avait voulue Comte et telle que l'a comprise Littré et ses proches.

La présentation de la Société, et de ses statuts, lui donne pour but «l'étude scientifique des problèmes sociaux et politiques » (art. I) $)^{34}$, et précise aussitôt l’allégeance à la «philosophie positive» (art. 2) ${ }^{35}$. Le lieu des séances (art. I2) «au local de la Société, I6 rue de Seine» souligne encore les liens avec la revue La Philosophie positive, puisque c'en est aussi le siège. Il y a 26 membres fondateurs, auxquels sont adjoints des correspondants étrangers; la plupart sont auteurs dans la revue de Littré et Wyrouboff ${ }^{6}$.

Les premiers mémoires lus en séance en disent long sur les tensions voire les dissentiments entre les conceptions de la sociologie comtienne et celles des sociétaires.

D’abord, si la classification des éléments de la sociologie a été discutée prioritairement, c'est bien que les programmes de Comte sont considérés insuffisants. Tous les intervenants le soutiennent avec plus ou moins de fermeté. Ainsi Grégoire Wyrouboff assure-t-il que la loi des trois états est une question «controversée encore » et il juge que Comte a bel et bien commis une «erreur» en intégrant dans la sociologie les études des facultés et sentiments de l'individu qui doivent relever de la psychologie (Wyrouboff, I872, p. 303). Joseph de Bagnaux rappelle «un fait au sujet duquel nous sommes tous d'accord, c'est que nous rejetons comme erronée une portion plus ou moins étendue des conclusions auxquelles ces vues ont conduit Auguste Comte sur le terrain de la sociologie» (Bagnaux, 1872, p. 317). Gustave Hubbard prend également des distances avec Comte $^{37}$. Et les propositions faites par les uns et les autres sont effectivement fort éloignées des positions comtiennes. Ainsi Wyrouboff, qui affirme s'appuyer sur «la séparation du savoir abstrait et du savoir concret» comme «notion indiscutable»

34 Littré, 1872a, p. 298 : le terme « scientifique » est souligné dans le texte.

35 Ibid., «Conformément aux principes propres à la philosophie positive, elle [la Société] admet que ses travaux doivent avoir exclusivement pour base l'examen des lois naturelles qui règlent la constitution et la marche de sociétés».

36 Voir les tableaux établis par Heilbron (2007).

37 «[T] out en admettant un très grand nombre de ses points de vue, je dois vous avouer que je ne saurais m'enfermer dans le cercle qu'il donne d'habitude à ses conceptions. 》) (Hubbard, |872, p. 33I). 
(Wyrouboff, 1872, p. 304), se demande pourtant aussitôt: «Devons-nous nous occuper exclusivement de la partie abstraite de la science sociale? » (ibid., p. 305). Il conteste aussi la manière dont Comte organisait la sociologie statique en études sur les «niveaux individu, famille, nation», puis «race» et «humanité», car, d'une part, il refuse le niveau individuel et, d’autre part, il ajoute un terme entre la famille et la nation, «celui des classes sociales», qui, ayant échappé à Comte l’aurait conduit, de façon erronée, à «rejet[er] en bloc toute l'économie politique» (ibid., p. 308) 38. De même, lorsque Wyrouboff décline la «dynamique sociale», il le fait en termes fort peu comtiens, demandant expressément qu'elle comporte «l'histoire des religions et des philosophies», l'étude du droit, et «ce qu’on appelle l'Économie politique et ce que je préfère appeler l'Économie sociale» (ibid., p. 310 et p. 312). Quant à Bagnaux, qui propose deux branches de la sociologie - «la théorie, et les objets d'application de la science» qu'un tableau joint regroupe sous la dénomination de «pratique» (Bagnaux, I872, p. 320-322) -, il subvertit la distinction à laquelle Comte tenait entre études abstraites et théoriques et études pratiques ou appliquées, la sociologie comme science fondamentale devant s'occuper des premières seulement. On retrouve chez Adolphe Clavel un pareil oubli du souci comtien de se garder des applications concrètes ${ }^{39}$. Et les divisions proposées par Hubbard (I872, p. 332-333) - $\mathrm{I}^{\mathrm{re}}$ division, «Étude des milieux ou Géographie»; $2^{\mathrm{e}}$ division,

38 Ce reproche d'avoir négligé l'économie politique avait déjà été fait par Littré (I863, p. 674-675) et par Jules Ferry qui, dans un des tout premiers numéros de La Philosophie positive, jugeait que l'«anathème» porté contre les économistes par Comte a pour «sens vrai» la «rénovation de l'économie politique» et que «rien n'est plus important [...] qu'un travail scientifique tendant à incorporer l'économie politique dans la science sociale. Il est impossible, au temps où nous sommes, de ne point réviser, interpréter ou compléter le jugement si bref d'Auguste Comte [qui a] exécuté l'économie politique en quelques pages.» (Ferry, 1867, p. 289-290).

39 Son mémoire confère à la sociologie « deux portions distinctes, l'une purement spéculative et traitant des équilibres organiques en vertu desquels existe la société; c'est la statique sociale dont le caractère général et abstrait ne doit pas être méconnu. [...]. L'autre portion de la sociologie passe du domaine de l'abstrait dans le domaine du concret, sort de la théorie pour arriver à la pratique et de l'équilibre pour arriver au mouvement.» (Clavel, 1872, p. 329). Mais pour Comte statique et dynamique sociales devaient toutes les deux relever de l'approche fondamentale, théorique et abstraite. De plus, Clavel insiste sur le rattachement de la sociologie à la biologie d'une manière qui contredit les efforts faits par Comte pour distinguer les deux sciences - ce que, par contre, Littré (1868) défendait. 
«Tableau des progrès de l'humanité ou Histoire» - rabattent la sociologie sur des sciences naturelles particulières desquelles Comte voulait justement la différencier.

Lorsque Littré à son tour donne plus de précisions sur la sociologie promue en proposant un «Plan d'un traité de sociologie», il expose, en dépit de l'allégeance comtienne déclarée ${ }^{40}$, des demandes qui sont clairement des modifications profondes. Certes, il défend fermement contre d’autres approches la spécificité de la sociologie et son autonomie ${ }^{41}$, et la définition donnée - «La sociologie est la science des faits et des lois qui régissent l'existence et le développement des sociétés » (Littré, I872b, p. I53) - paraît très orthodoxe. Mais il s'empresse de rapporter une objection contre l'idée comtienne de traiter la statique avant la dynamique, et déclare aussitôt «qu'il ma été plus commode de placer l'état dynamique le premier» (ibid., p. I54-I55). Il adopte donc cet ordre inversé et crée toute une série de néologismes, appuyés sur l'étymologie, pour définir les parties successives. Ainsi la dynamique, devenue sociodynamie, précède la statique devenue sociomérie. - Pour la sociodynamie, où il distingue «l'état dynamique d'entretien et l'état dynamique de progrès », Littré propose les termes de sociergie et sociauxie, la première devant être traitée prioritairement. La sociergie porte sur cinq sujets : le gouvernement, la production matérielle, la culture de la morale, celle des beaux-arts et celle des sciences, et pour chacune des spécialités qui en découlent, qu'il décline sous de nouveaux noms ${ }^{42}$, Littré insiste sur le renvoi à l’histoire (ibid., p. 156-157). Pour la sociauxie ou état dynamique d'évolution, où Littré défend les nécessaires appuis sur des documents historiques, il reprend certes un peu les «états» définis par Comte, mais le développement en huit chapitres suggère une nette correction de la logique de succession des trois états (ibid., p. I57-I59) ${ }^{43}$. - Pour la sociomérie, où Littré voit deux parties «suivant

40 Littré y fait état (p. 153-154) d'un projet de rédaction de «six traités représentant les six sciences abstraites dans l'ordre hiérarchique déterminé par M. Comte», projet partagé avec Robin et Wyrouboff. Littré devait se charger de l'ouvrage sur la sociologie, car, répète-t-il, «il n'existe point de traité de sociologie». Si Comte en est donné pour l'«immortel fondateur», il est aussi dit n'en avoir établi que «les préalables».

41 Question centrale de Littré, 1868.

42 Respectivement: sociarchie, socioporie, sociagathie, sociocalie et socialéthie.

43 Les huit chapitres concernent «l'accumulation en général, l'état empirique, l'état théologique, le génie de la poésie et des arts, l'état positif, les milieux et les races». 
qu'elle se rapporte à l'état dynamique d'entretien ou à l'état dynamique d'évolution», il dit aussi qu'il «intercale le chapitre sur la démographie, fort important aussi et élaboré indépendamment sous le nom de statistique» (ibid., p. 159); or ceci va à l'encontre des méfiances de Comte envers le recours aux statistiques. Et pour les études des différents groupements, Littré corrige encore la manière dont Comte en établissait la succession ${ }^{44}$. Enfin Littré propose une autre partie consacrée aux études des troubles sociaux et nommée sociotaraxie, qu’il partage en «quatre chapitres, selon que les troubles se rapportent à l'état statique, à l'état dynamique d’entretien, au fortuit dans l'histoire, à l'état dynamique d'évolution» (ibid., p. I59-160).

Quant à la pratique «sociologique» de Littré, c'est aux articles réunis dans Fragments de philosophie positive et de sociologie contemporaine, qu'il faut se reporter. Recueillis selon l'ordre chronologique, ces textes traitent de sujets très divers, et plutôt bien délimités et particularisés. Difficile de trouver dans cette hétérogénéité le souci de visions d'ensemble voulues par Comte pour sa sociologie; la sociologie «fragmentée » de Littré n’a plus grand-chose à voir avec les ambitions systématiques de Comte, qui en aurait condamné le mode «dispersif». Dans le recueil de Littré, le trait le plus commun des articles est toujours l'histoire: plus de la moitié ont des titres qui les donnent explicitement pour des travaux historiques, et l'histoire a bonne part dans les autres ${ }^{45}$. Les mêmes commentaires sont à faire sur les publications ultérieures de Littré. Ainsi pour les «Remarques» ajoutées à la deuxième édition de Conservation, Révolution et Positivisme. Littré déplore «maintes erreurs et maints faux jugements» dans son adhésion hâtive aux vues comtiennes et se reproche tout particulièrement d'avoir versé dans l'«utopie», confondant «prévision», d’une part, et «hypothèse», «prophétie» ou «conjecture», de l'autre (1979a, p.64, p. I36, p. 289-292, p. 328, p. 365-366, p. 484). Bref, si Littré garde la

44 Pour Littré, «le premier groupe est la tribu ou le clan; historiquement on ne remonte qu'à la tribu et non à la famille». Puis «après la tribu vient la cité ou la commune, puis la nation, puis [...] les groupes de nations appartenant à un même système de civilisation; enfin l'humanité en perspective, comme l'a établi M. Comte». Littré revendique aussi d'avoir «le premier distingué [...] l'échelon que je nomme système de nations». (ibid., p. I59).

45 «Du développement historique de la logique », «Du progrès de la science et de la philosophie depuis le commencement du siècle », «Du progrès dans les sociétés et dans l'État, etc., ou encore: «Histoire de la révolution de |848 », «Études sur la crise de guerre de 1870 et $|87|$ », etc. 
philosophie de l'histoire de Comte d’après son schéma général jugé si «admirablement exposé» (ibid., p. 6I), il est clair qu'il ne souscrit plus à la même sociologie. En I880, le dernier gros recueil de Littré, De l'établissement de la Troisième République, montre combien sa sociologie bascule dans l'analyse de l'histoire politique ${ }^{46}$.

Heilbron a donc eu raison de souligner que ce qu'il appelle une «reconceptualisation» (Heilbron, 2007, p. 318) de la sociologie par Littré la transforme en une sorte de commentaire de la situation politique française et en «plaidoyer pour une pratique politique plus "modeste”, plus circonstanciée» (ibid., p. 321) ${ }^{47}$. Et sans doute aussi lorsqu'il attribue l'échec de l'éphémère Société de sociologie au fait qu'elle fut fondée pour «remplir une fonction avant tout politique», tout en soulignant qu'«il ne faut pas pour autant surestimer la cohérence politique du groupe» (ibid., p. 322-326). Faut-il cependant aller jusqu’à dire que la Société de sociologie a «avant tout été créée comme un club politique » et lui dénier des ambitions de société savante (ibid., p. 326-327) ? C’est manquer dans le travail de Littré, son attitude décidément historienne, son souci d’analyste précis des situations historiques, des conditions différentielles qu'il s'applique à mettre à jour, afin d'éviter ce qu'il reproche à Comte - les grandes questions et fresques générales et les prévisions basculant dans l'utopie; c'est laisser échapper ce qui fait la richesse et l'originalité de la pratique scientifique de Littré, celle d'un intellectuel érudit attentif aux détails et aux cas - ce que Comte d’ailleurs lui reprochait durement. En sociologie comme ailleurs, Littré s'est avant tout voulu historien ${ }^{48}$.

Il me semble que l'on peut alors s'interroger différemment sur la postérité de la sociologie littréenne. Sans doute Heilbron a-t-il encore raison en identifiant Littré et les positivistes hétérodoxes comme la cible de certains jugements très critiques de Durkheim contre des sociologues non nommés ${ }^{49}$. Mais pour éclairer

46 Voir aussi Littré, 1879b - petite brochure.

47 De même, les tournures de «sociologie pratique» ou de «sociologie contemporaine», dont Littré se réclame expressément, sont soulignées avec pertinence, voir p. 322, p 327 et p. 328.

48 La même conclusion convient pour ce qui est de l'apport de Littré aux questions philologiques et médicales. Voir Petit, 1982 et 2014.

49 Voir Heilbron 2007, p. 329, renvoyant à un article de Durkheim de 1895 sur «l'état actuel des études sociologiques en France». II y a des critiques du même type dans les Règles de la méthode sociologique: selon Philippe Steiner, 1995, la cible qui est alors un «historien» 
l'histoire de la sociologie, s'il est nécessaire de rappeler le rôle de Littré et de la première Société de sociologie ainsi que de bien préciser les nets décalages entre les conceptions et pratiques littréennes et celles de Comte, il serait aussi utile, pardelà l'analyse de leur «échec» et de leur «rejet» (Heilbron, 2007, p. 329), de se demander si ces sociologies balbutiantes n’ont pas eu un rôle constructif par-delà celui de repoussoir que leur a fait jouer l'entreprise d'emprise des durkheimiens sur la sociologie. Or, à la fin du XIxe siècle et au début du Xxe, il existe une sociologie elle aussi un peu trop oubliée: celle qui se fait autour de René Worms de son Institut international de sociologie et de sa Revue internationale de sociologie fondés en 1893 et elle est dominante avant que Durkheim et son école en envahisse le champ ${ }^{50}$. Cette sociologie fait plutôt bon accueil aux positivistes, qui, de leur côté, ont dépassé les luttes exaspérées entre orthodoxes et hétérodoxes et se retrouvent dans le mouvement très élargi de la Société positiviste internationale et sa très ouverte Revue positiviste internationale. Trois membres de la nouvelle Société de Sociologie de Paris l'étaient de la première Société de sociologie et une trentaine d'autres membres et auteurs de la Revue sociologique internationale sont expressément liés aux institutions positivistes. Celles-ci font d’ailleurs volontiers et régulièrement de la publicité pour les cours et conférences de Worms.

L’analyse des liens entre les conceptions et les institutions sociologiques de ces mouvements, dont les succès du durkheimisme ont estompé l'importance, est à approfondir.

\section{BIBLIOGRAPHIE}

AUDERN F. et BORLANDI M. (dir.), 2015, «La sociologie de René Worms (18691926)», Les Études sociales, 161-162.

BAGNAUX J. de, 1872, «Mémoire sur la division de la Société en sections 》, La Philosophie positive, VIII-I, p. 3I3-323.

générique, renvoie à Comte; mais Steiner montre fort bien que Durkheim critique en même temps le renvoi trop général à une philosophie de l'histoire et l'approche monographique, ce qui nous semble plutôt cibler effectivement une sociologie à la Littré.

50 Voir sur ce sujet les travaux réunis par Frédéric Audern et Massimo Borlandi, 2015. 
CLAVEL A., 1872, «Mémoire de M. le Dr Clavel, lu à la séance du Jeudi 8 février |872», La Philosophie positive, VIII-I, p.323-330.

COMTE A., 1969-1970 [1851-1854], Système de politique positive, 4 vol., Paris, Anthropos.

-, 1973-1990, Correspondance générale et Confessions, 8 t., Paris, Éditions de I'EHESS \& Vrin.

FERRY J., I867, «Marcel Roulleaux», La Philosophie positive, I-2, p. 289-312.

HEILBRON J., 2007, «Sociologie et positivisme en France au XIXe siècle: les vicissitudes de la Société de sociologie (1872-1874)», Revue française de sociologie, 48-2, p. 307-331.

HUBBARD G., I872, «Mémoire de G. Hubbard», La Philosophie positive, VIII-I, p. 33I-336.

LITTRÉ É., 1845a, De la philosophie positive, Paris, Ladrange.

-, I845b, Analyse raisonnée du cours de philosophie positive de M. Auguste Comte, Paris, Ladrange.

-, 1852, Conservation, Révolution et Positivisme, Paris, Ladrange.

-, 1859, Paroles de philosophie positive, Paris, Delahaye.

-, 1863, Auguste Comte et la philosophie positive, Paris, Hachette.

-, 1863-1873, Dictionnaire de la langue française, 4 vol., Paris, L. Hachette.

-, 1864 «Préface d'un disciple», in A. Comte, Cours de philosophie positive, Paris, Baillière, vol. I, p. V-L.

-, 1867, Auguste Comte et Stuart Mill, Paris, Baillière.

-, 1868, «De la condition essentielle qui sépare la sociologie de la biologie», La Philosophie positive, II-5, p. 186-207.

-, 1870, «De la méthode en sociologie», La Philosophie positive, VI-5, p. 29I-30I.

-, I872a, «Fondation de la Société de sociologie», La Philosophie positive, VIII-5, p. 298-301.

-, I872b, «Plan d'un traité de sociologie. Mémoire lu à la Société de sociologie le 23 mai |872», La Philosophie positive, IX-I, p. I53-I63.

-, 1873, La Science au point de vue philosophique, Paris, Didier.

-, 1876, Fragments de philosophie positive et de sociologie contemporaine, Paris, Bureaux de la Philosophie positive. 
-, 1877, «Étude sur les progrès du positivisme», La Philosophie positive, XVIII-4, p. 107-119.

-, 1879a, Conservation, Révolution, Positivisme, Paris, Bureaux de la Philosophie positive.

-, 1879b, Par quelle conduite la République française peut-elle consolider le succès qu'elle a obtenu?, Paris, Charavay.

-, 1880, De l'établissement de la Troisième République, Paris, Bureaux de la Philosophie positive.

PETIT A., 1982, «Philologie et Philosophie de l'histoire chez É. Littré», Revue de Synthèse, CIII-106/107, p. 215-243.

-, 1987, «La fin positiviste de la Révolution», in La Légende de la Révolution, S. Bernard-Griffiths (dir.), Clermont-Ferrand, ADOSA, p. 499-540.

-, 1992a, «Comte et Littré: les débats autour de la sociologie positiviste», Communications, 54, p. 15-37.

-, 1992b, «La réinterprétation positiviste de la Révolution par Émile Littré», in Le xIx Siècle et la Révolution française, A. Faure (dir.), Paris, Créaphis, p. 3II-327.

-, 1993, Heurs et malheurs du positivisme comtien. Philosophie des sciences et politique scientifique chez Auguste Comte et ses premiers disciples, 3 vol., Thèse de Doctorat d'État, Université Paris I.

-, 2014, «Émile Littré, médecin, philologue, historien », in Médecine, sciences de la vie et littérature en France et en Europe, de la Révolution à nos jours, L. DumasyQueffélec et H. Spengler (dir.), vol. 3, Le Médecin entre savoirs et pouvoirs, Genève, Droz, p. 343- 356.

PICKERING M., 1993-2009, Auguste Comte. An Intellectual Biography, 3 vol., Cambridge, Cambridge University Press.

REY A., 1970, Littré: I'humaniste et les mots, Paris, Gallimard.

ROL C., 20I5, «La Société de sociologie de Paris: un continent méconnu (1895-1952)», Les Études sociales, 161-162, p. 119-173.

WORMS R., 1910, «La première Société de sociologie (1872)», Revue internationale de sociologie, 18-2, p.202-206.

WYROUBOFF G., 1872, «De la classification de la sociologie», La Philosophie positive, VIII-I, p. 302-3I3. 